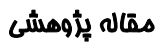

\section{جذب MTBE از محلولهاى آبى توسط زئوليتهاى اصلاح شده با سورفكتانتها}

\author{
رامين نبى زاده '، اميرحسين محوى '"، سيدكمال غديرى"، سيمين ناصرى"، عليرضا مصداقى نيا'، احسان ابويى مهريزى ه
}

'دانشيار كروه مهندسى بهداشت محيط، دانشكاه علوم يزشكى تهران، تهران، ايران

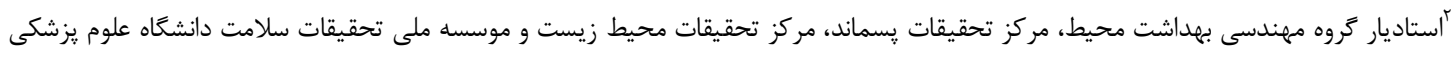
تهران، تهران، ايران

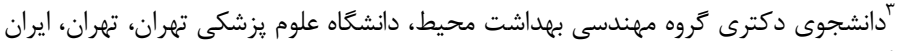

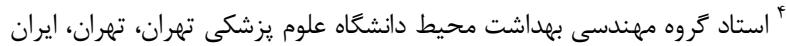

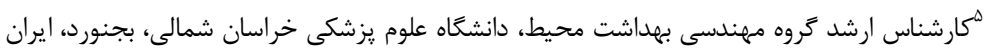

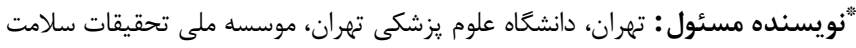
يست الكترونيك: ahmahvi@yahoo.com

وصول:r/rq/q/V:

جكيده

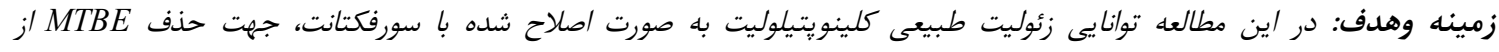

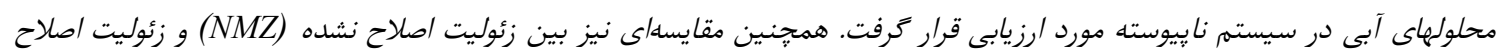

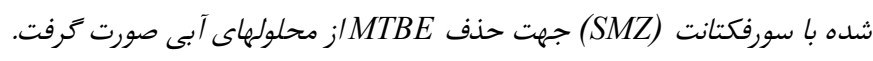

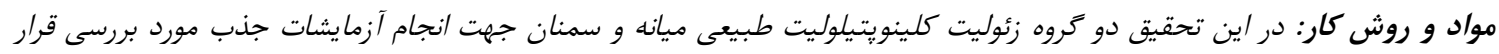

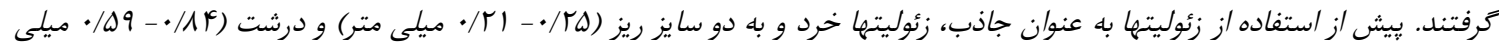

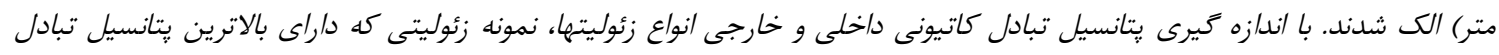

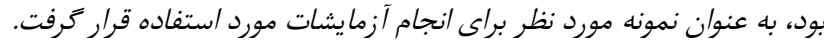

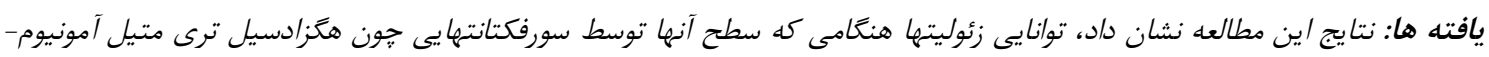

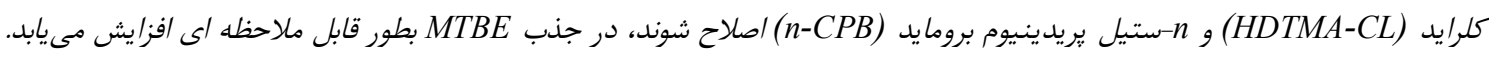

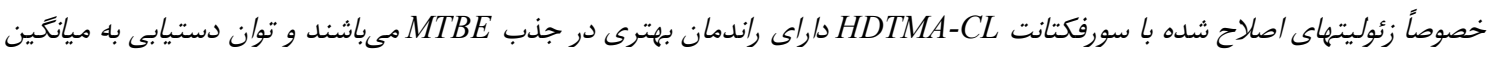

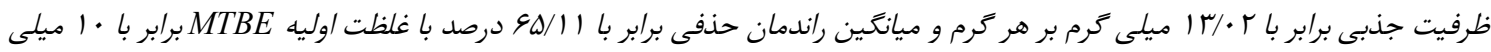

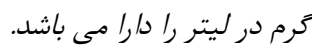

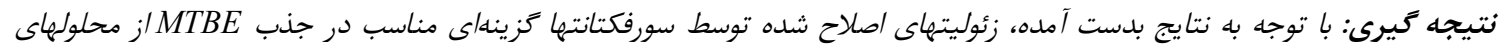
آبى مى باشند. وازه هاى كليدى: كلينويتيلوليت، زئوليت اصلاح شده، سورفكتانت، MTBE، جذب

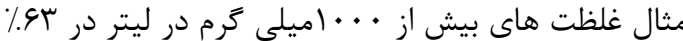

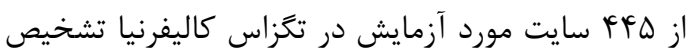

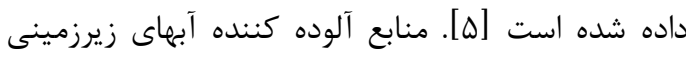

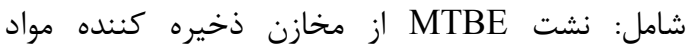
سوختنى، روان آبهاى سطحى و كشتى هاى نفتكش و وخدان

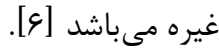

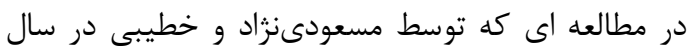

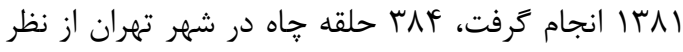
حضور MTBE مورد بررسى قرار كرفتند. نتايج اين

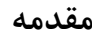

متيل ترشيرى بوتيل اتر (MTBE) تركيبى است كه از

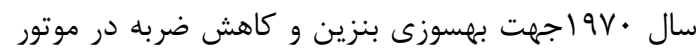

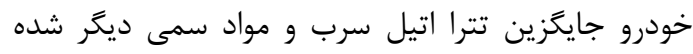

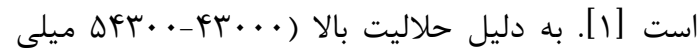

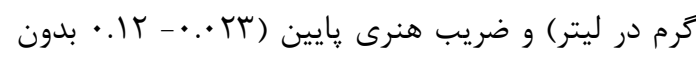

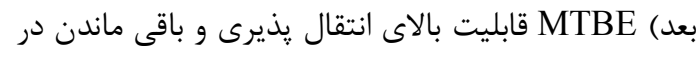

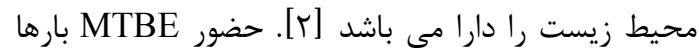

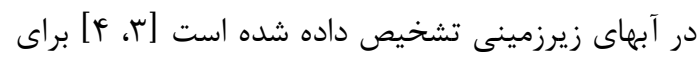


تركيبات جانبى در آب آشاميدينى طى فرايندهاى جذب، اين روش را به يكى از مطلوبترين روش هاى مورد توجه

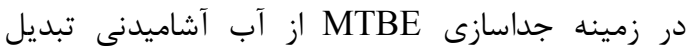

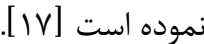

زئوليتها مواد معدنى طبيعى مى باشند كه داراى ظرفيت

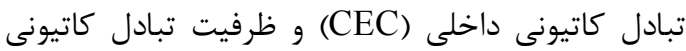

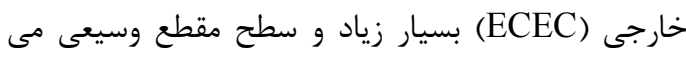

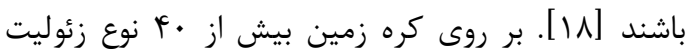

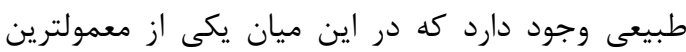

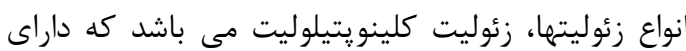

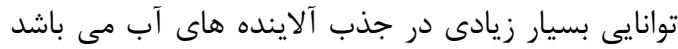
[19، •r، [r]]. نتايج تحقيقات بسيارى نشان ميدهدكه

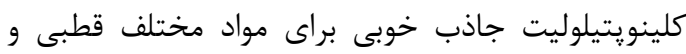
غير قطبى مانند هيدروكربنهاى آليفاتيك و آروماتيك، داديك الكلها، كتونها و موادى جون , و ساير مواد مى $\mathrm{H}_{2} \mathrm{~S}, \mathrm{NH}_{3}, \mathrm{Cd}, \mathrm{Cs}, \mathrm{Sr}, \mathrm{Mn}, \mathrm{As}, \mathrm{Cr}$

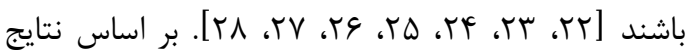
بدست آمده از مطالعات قبلى، زئوليتهايى كه داراى

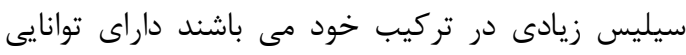
جذب MTBE بيشترى نسبت به كربن فعال مى باشند

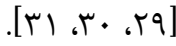

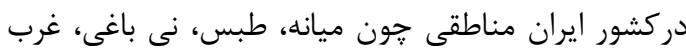

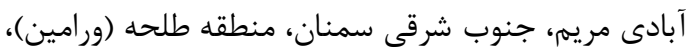

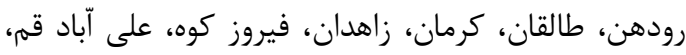

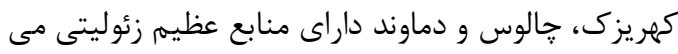
باشند كه مى توان از آن به عنوان سرمايه عظيم ملى نام دام

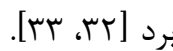

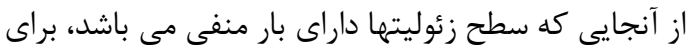

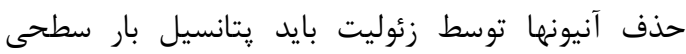

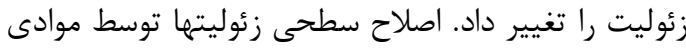

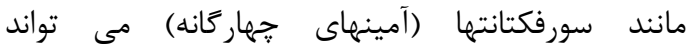
مشخصات سطحى زئوليتها را تغيير داده و زئوليتها را بهان البهان

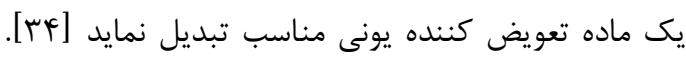
سورفكتانتها مى توانند بر روى سطح خارجى زئوليت يك نائن

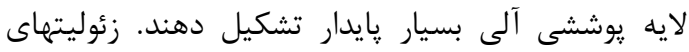

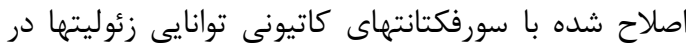
جذب آنيونها (نيترات، فسفات، آرسنات، كرومات و غيره)،
مطالعه حاكى از اين مطلب بود كه، مقادير قابل اندازه كيرى از MTBE در شش خاه از اين منطق آنه وجود داشت

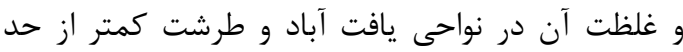

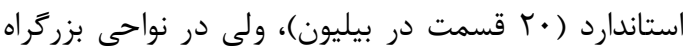
آيت الله سعيدى و شهرى شريعتى بالاتر از حد استاندارد

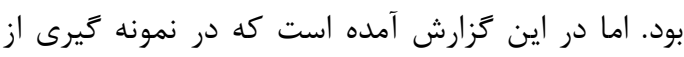

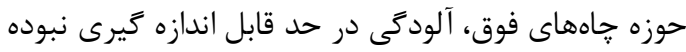

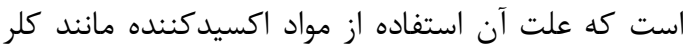

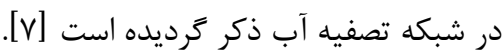
در مطالعهى ديخرى كه در ايران انجام كرفته است، در دردئه

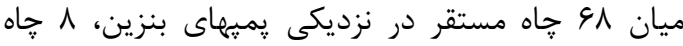

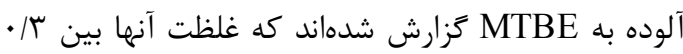

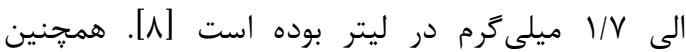
اسلامى و همكاران، غلظت MTBE در نمونههاى آب درائ زهكشى شده از جايكاه هاى سوخت شهر زنجان ران ران دان در

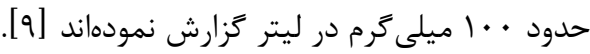
مهمترين دلايلى كه باعث مىشوند MTBE به يكى عامل زيست محيطى مهم تبديل شود شامل يتانسيل

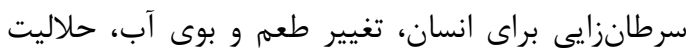

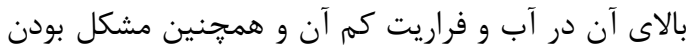

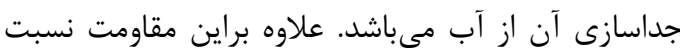

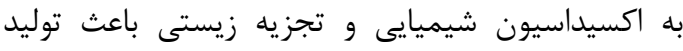

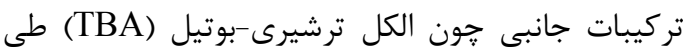

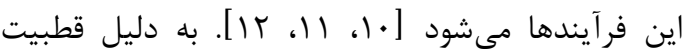

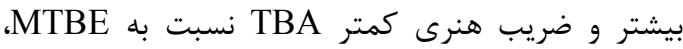
حذف TBA از آب نسبت به حذف MTBE مشكل تر

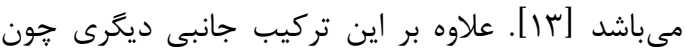

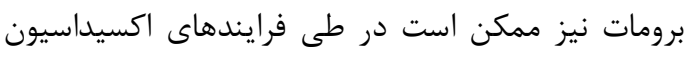

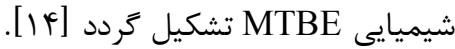

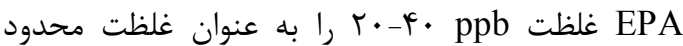

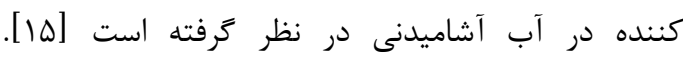
همجنين ديارتمان خدمات بهداشتى كاليفرنيا نيز ميزان

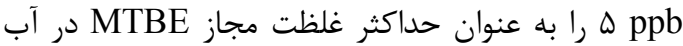

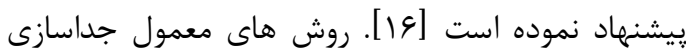
MTBE

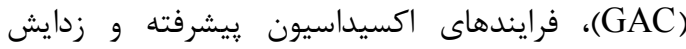
توسط تزريق هوا به داخل آب مى باشد. عدم توليد 
غلظت برابر mmol/L و · CMC به عنوان غلظت

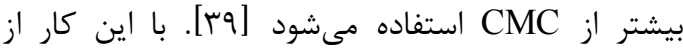

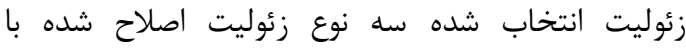

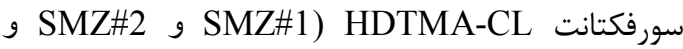
n- و سه نوع زئوليت اصلاح شده با سورفكتانت (SMZ\#3 و و SMZ\#4) CPB زئوليت اصلاح نشده (NMZ) كه در مجموع هفت نوع

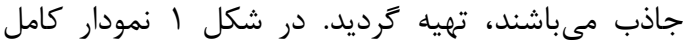
مراحل توليد زئوليتهاى اصلاح شده توسط سورفكتانتها ترائ

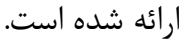

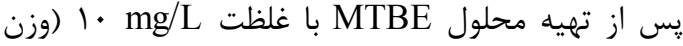

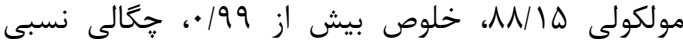

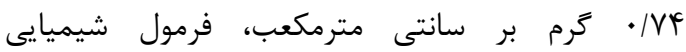

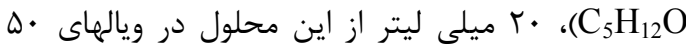

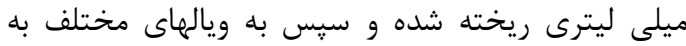
ميزان r/ • كرم از هر نوع جاذب توليد شده اضافه كرديد.

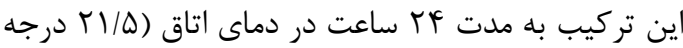

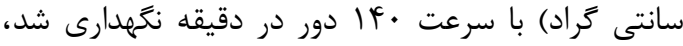
شرايطى كه براى به تعادل رسيدن مناسب تشخيص داده

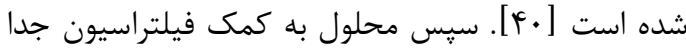
شده و غلظت MTBE باقيمانده توسط دستخاه

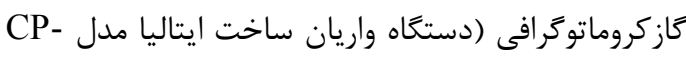

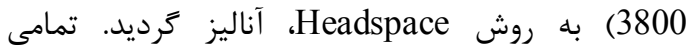
آزمايشات دو بار تكرار شده و ميانگين آن مورد تحليل قرار

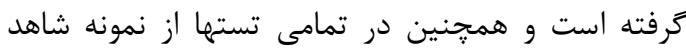

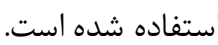
در نهايت از ميان جاذبهاى مختلف، جاذبى كه بيشترين

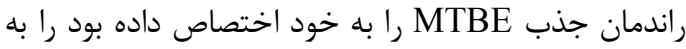
عنوان جاذب نمونه براى انجام ادامه تحقيق انتخاب شد. به منظور بررسى نسبت جاذب به محلول بهينه، از ميان

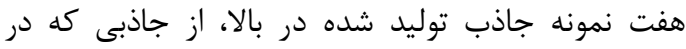

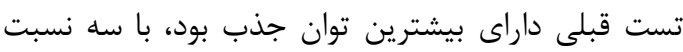

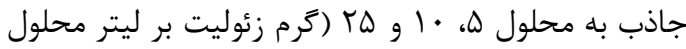

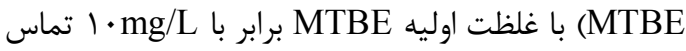

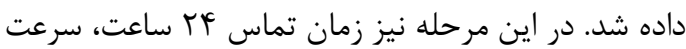

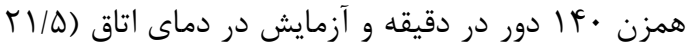
درجه سانتى كراد) انجام كرفت.
1) محلولهاى آلى غيرقطبى و هيدروكربن هاى آروماتيك نسبت به زئوليتهاى اصلاح نشده افزايش مى دهئ دهند [هـائ. در اين تحقيق، توانايى جذب MTBE توسط زئوليتهاى

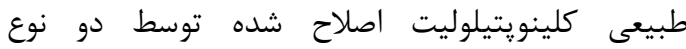
سورفكتانت (HDTMA-CL و CPB) مورد بررسى قرار كرفته است. حذف MTBE به دليل تاثيرات اين ماده

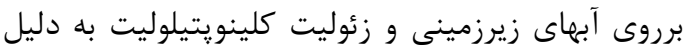
توانايى بالا در جذب آلاينده هاى محلول در آب انتخاب

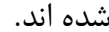

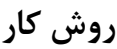
در اين مطالعه از دو نوع زئوليت تهيه شده از مناطق ميانه

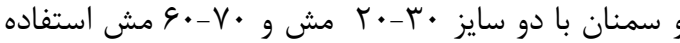
شد. CEC و ECEC اين جهار كروه زئوليت (كه بيان

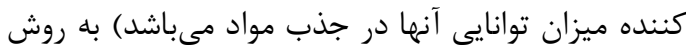

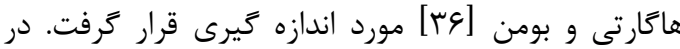
ميان انواع زئوليتهاى ذكر شده زئوليتى كه داراى بيشترين ECEC و CEC MTBE

زئوليت منتخب جهت تشخيص نوع كريستالينيته و مقايسه با زئوليت كلينويتيلوليت مرجع، توسط تست (دستخاه يراش يرتو ايكس از شركت شيمادزو مدل

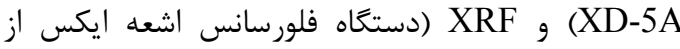
شركت آكسفورد مدل ED2000) مورد آناليز قراركرفت. جهت تهيه جاذب اصلاح شده، يس از شستشوى زئوليت

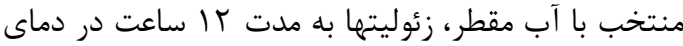
.

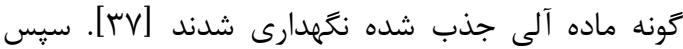

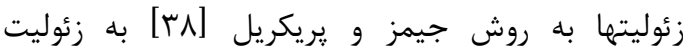

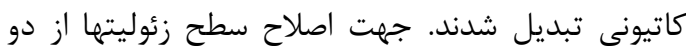
نوع سورفكتانت با نام هاى هكزا دسيل ترى مترئ متيل

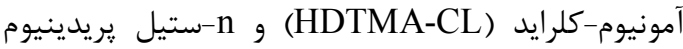
برومايد (n-CPB) استفاده شد. تمامى مواد مورد استفاده در اين تحقيق از شركت مرك خريدارى شد. جهت اصلاح

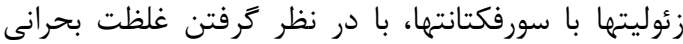

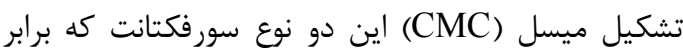

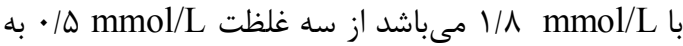

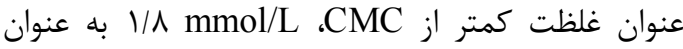




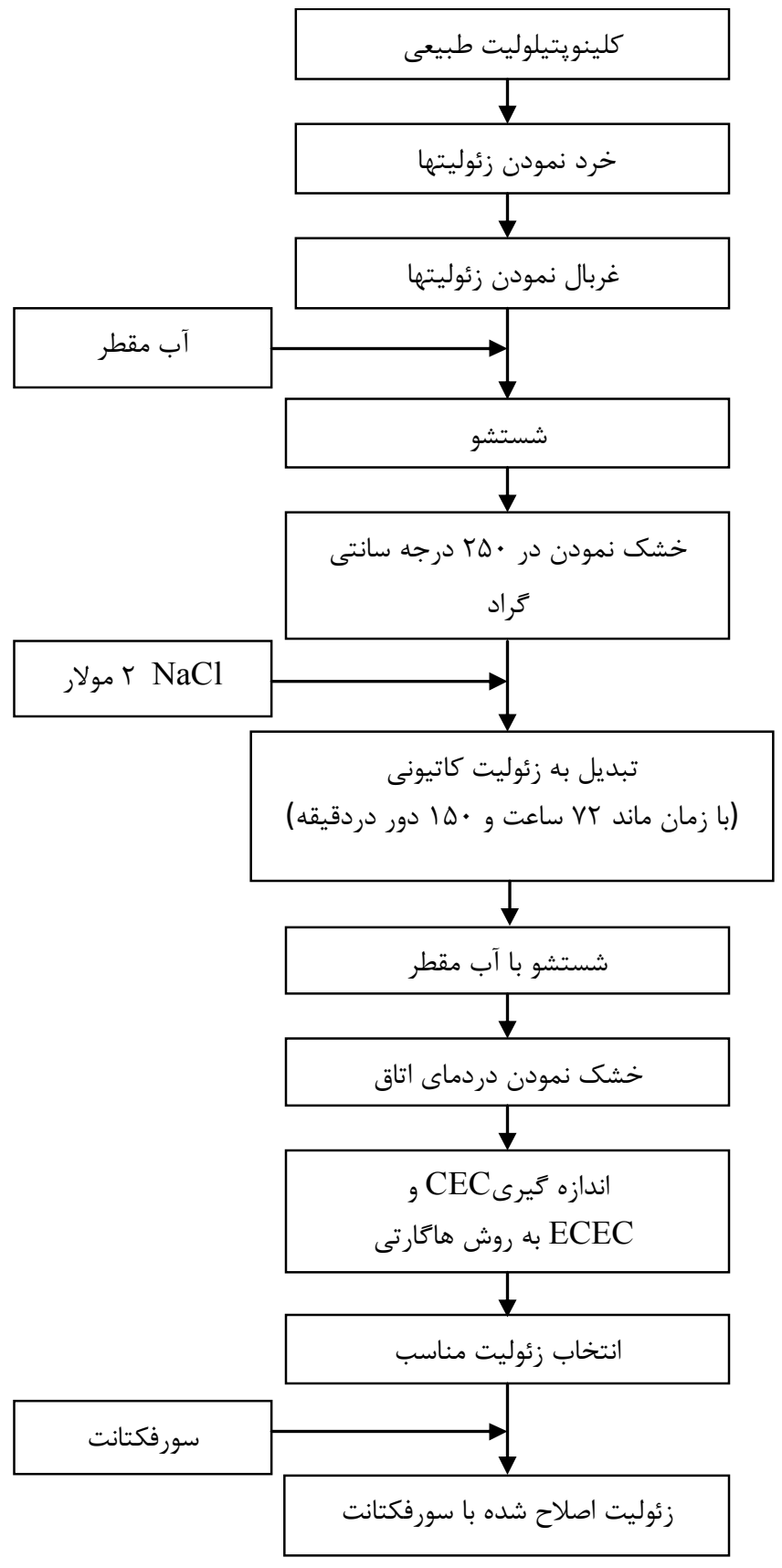

شكل ا: مر احل آماده كردر، زئوليتهاى اصلاح شده با سور فكتانت (SMZS)

(ساير شرايط مشابه تست قبل بود). راندمان و ظرفيت

جذب به ترتيب توسط فرمولهاى زير محاسبه كرديد:

$E=\frac{\left(C_{i n}-C_{t}\right) \times 100}{C_{i n}}$

$q=\frac{\left(C_{i n}-C_{t}\right) \times V}{W}$
در ادامه مراحل آزمايشات، از بهترين جاذب انتخاب شده و با نسبت جاذب به محلول بهينه براى به دست آوردن آداني

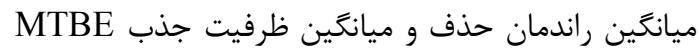

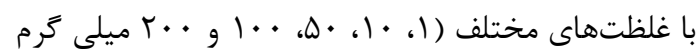

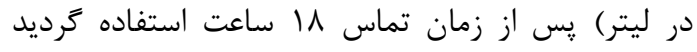


تست XRD انجام كرفته بر روى زئوليت ميانه كه الكوى آن در شكل r نشان داده شده است بيان مىنمايد كه جنس اين زئوليت از نوع كلينويتيلوليت مى باشد. همجنين إنين

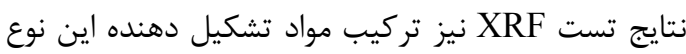

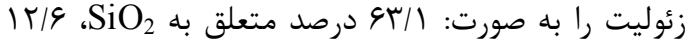
درصد متعلق به

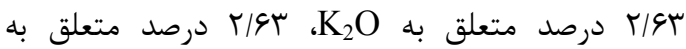

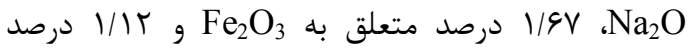
متعلق به MgO كزارش مىنمايد.

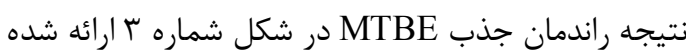
است. درميان زئوليتهاى اصلاح شده با دو نوع سورفكتانت ) و يك نمونه زئوليت اصلاح نشده HDTMA-CL NMZ)، زئوليتى كه توسط سورفكانت ئل

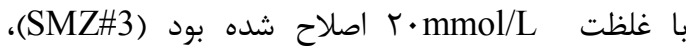
بالاترين توان جذب MTBE (حدود V^ درصد) را از خود

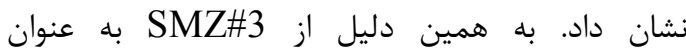
مناسبترين جاذب در آزمايشات بعدى استفاده كرديد. نتايج جذب MTBE توسط نسبتهاى مختلف جاذب به إنهائ

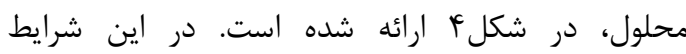

كه در اين فرمولها E راندمان جذب MTBE بر حسب

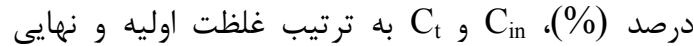

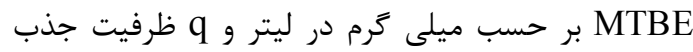

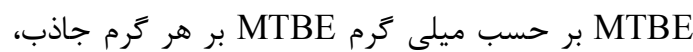

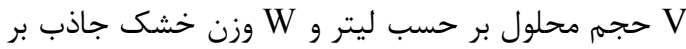
حسب كرم مى مجاشد. تمامى تستها به منظور كاهش خطا دو باشد بار انجام كرفت و نتيجه اعلام شده ميانكين دو تست انجام كرفته مىباشد و از نمونه هاى شاهد نيز براى بررسى تاثير عوامل مختل

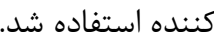

يافته ها بررسى ECEC و دو نوع زئوليت كلينويتيلوليت

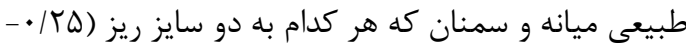

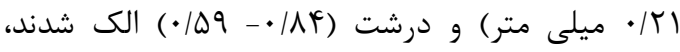
نشان داد كه زئوليت ريز ميانه داراى بيشترين ظرفيت داريت

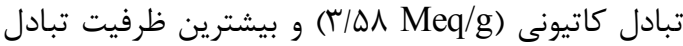

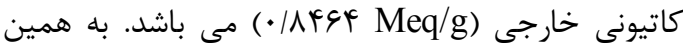

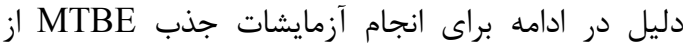

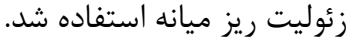

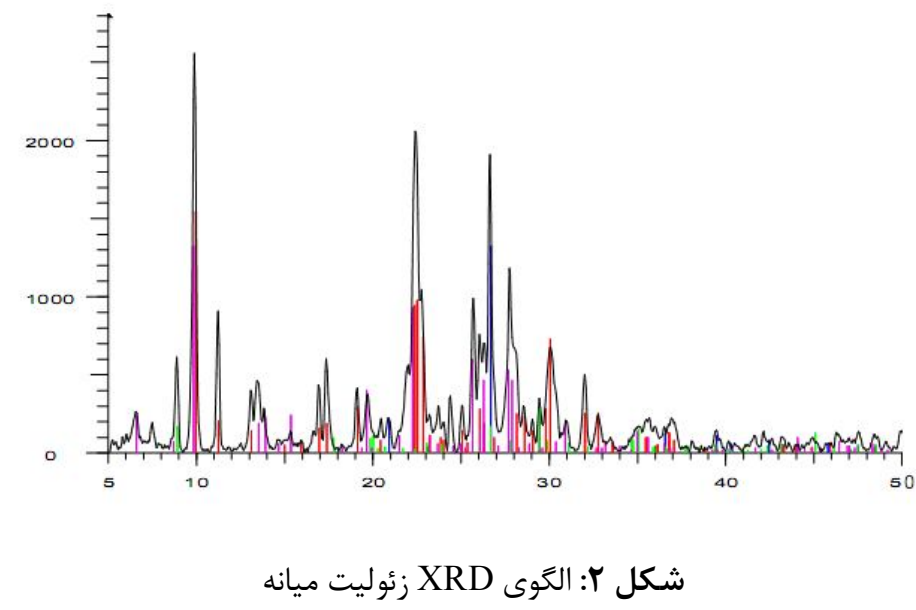




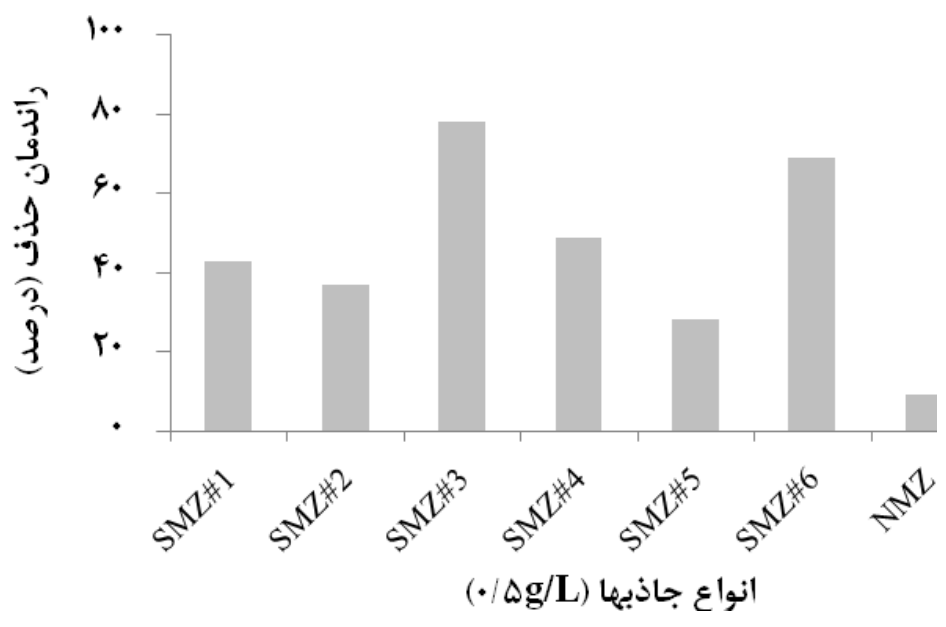

شكل r: راندمان حذف MTBE توسط جاذبهاى مختلف (غلظت اوليه lom/L MTBE)

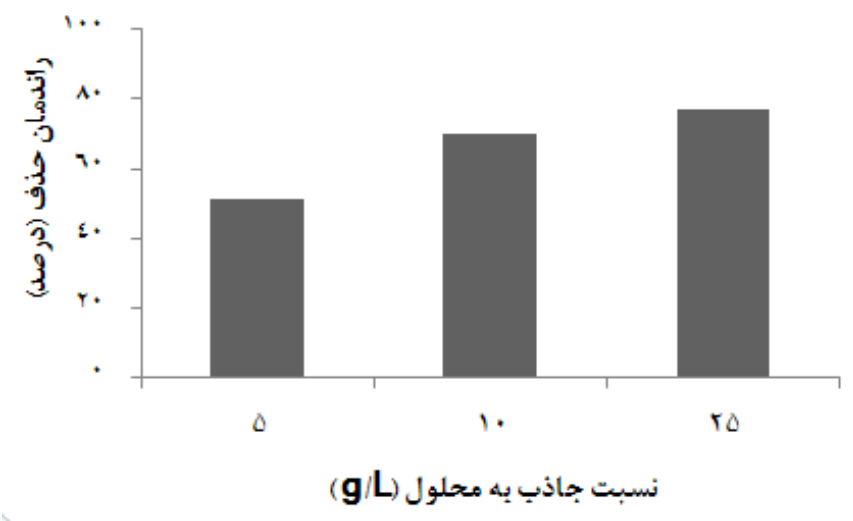

شكل \&\&: راندمان حذف MTBE با نسبتهاى مختلف جاذب به محلول

( ) ( اولظت اوليه mg/L MTBE) 


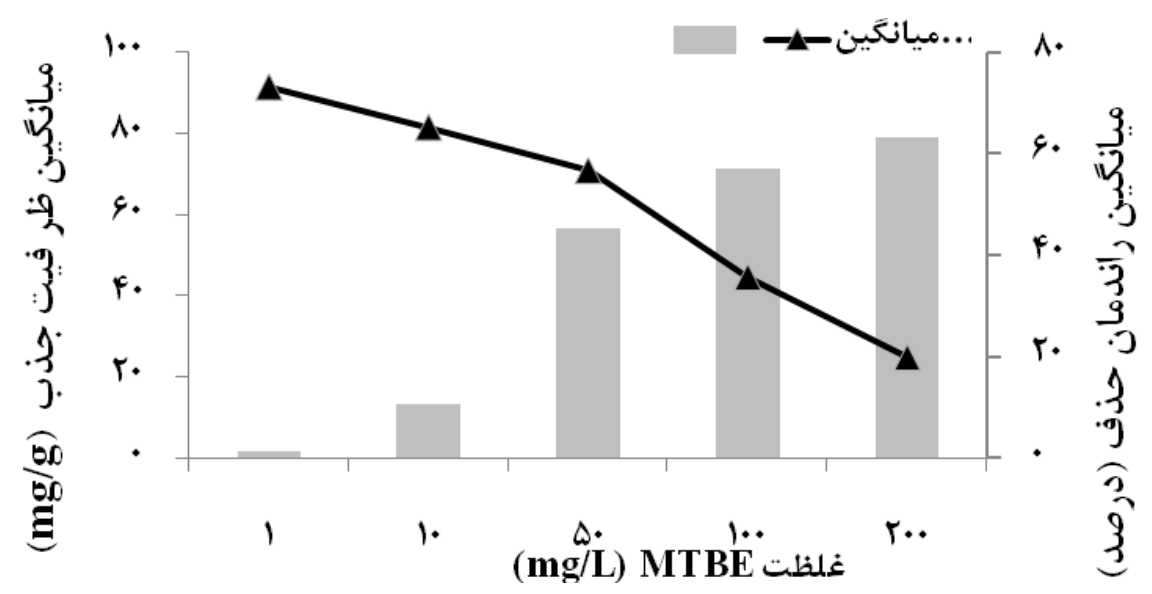

شكل ه: ميانگين راندمان حذف و ظرفيت جذب MTBE توسط جاذب SMZ\#3 يس از زمان تماس \\ ساعت

نتايج حاصله نشان مىدهد زئوليتهاى اصلاح شده با

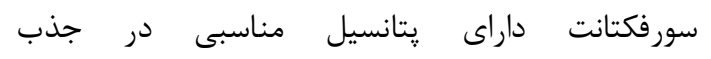

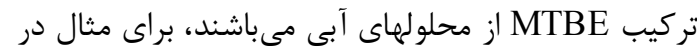
شكل ب زئوليتهاى اصلاح شده با سورفكتانت

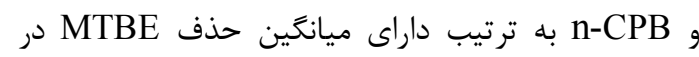

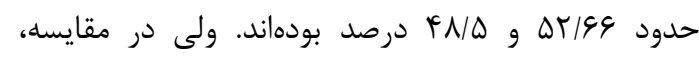

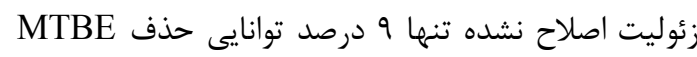
را دارا مىباشد. در مقايسه بين دو نوع سورفكتانت

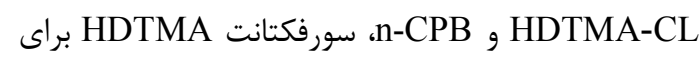
اين منظور بهتر عمل مئمايد كه اختلاف در فرمول

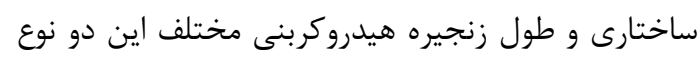

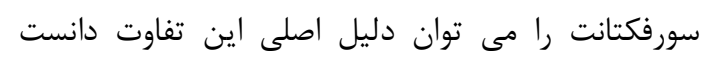

.[F.]

در جدول | ظرفيت جذب MTBE توسط جاذبهاى

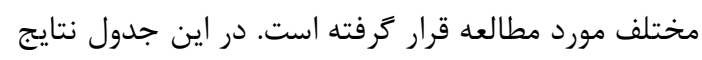

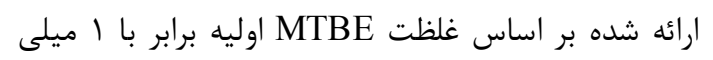
كرم بر ليتر مىباشند. همجنين نتايج اين تحقيق بيان مىنمايند كه اصلاح سطحى زئوليت كلينويتيلوليت با غلظت سورفكتانت بين بيشت بـان

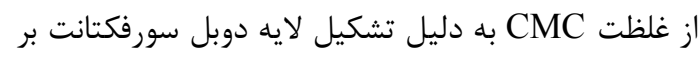

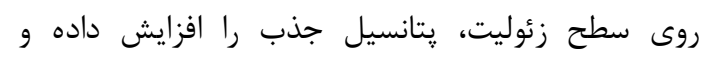

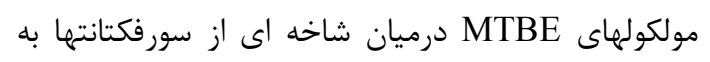

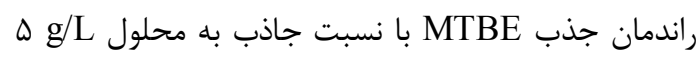

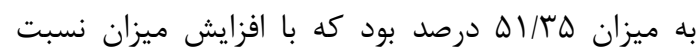

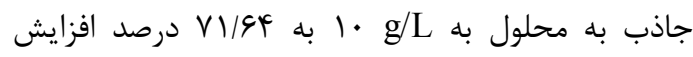

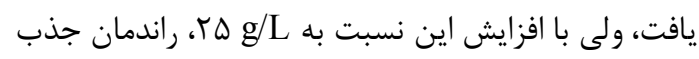

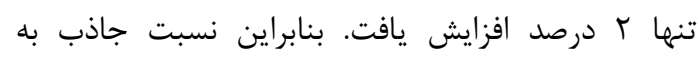

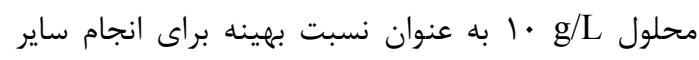
تستها انتخاب شد. در شكل شماره ه ميانگين راندمان حذف و ميانگين ظرفيت جذب MTBE در غلظتهاى مختلف توسط جاذب مانب SMZ\#3 ليتر) الرائه شده است. سنجش پارامترهاى ECEC و CEC جهار نمونه زئوليت بيان كننده اين واقعيت مى باشد كه بارئ كاهش سايز

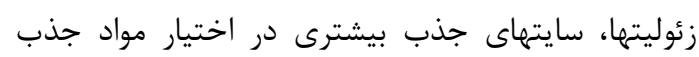

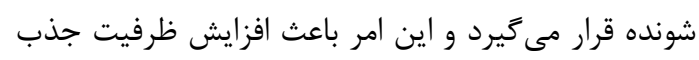

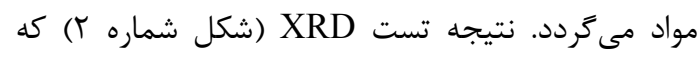

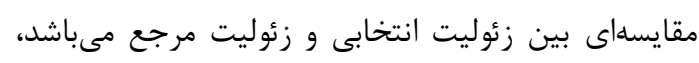

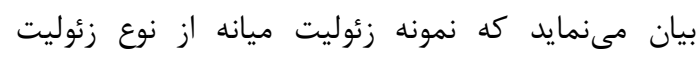

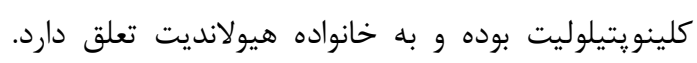

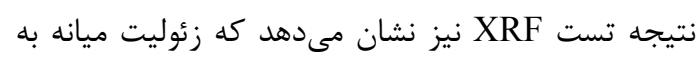

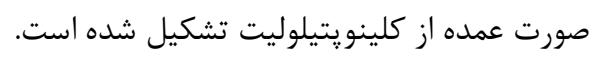




\begin{tabular}{|c|c|c|c|}
\hline 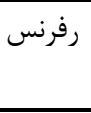 & 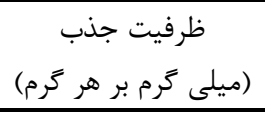 & رنج غلظت اوليه مطالعه شده & 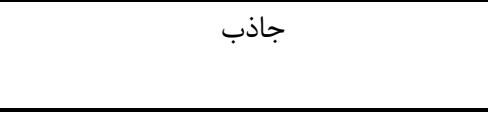 \\
\hline$\psi_{1}$ & $r r / F$ & $\cdot / 11-9 \vee / r$ & آمبرزورب بوه (نوعى جاذب سنتتيك) \\
\hline kt & $10 / 9$ & $\cdot \mid q-r \Delta \cdot \cdot$ & آمبرزورب بوه (نوعى جاذب سنتتيك) \\
\hline kt & N/9 & $1-r \cdots$ & آمبرزورب QVY (نوعى جاذب سنتتيك) \\
\hline il & $1 T / 9$ & $\cdot / 11 r-1 \cdot r$ & آمبرزورب بوه (نوعى جاذب سنتتيك) \\
\hline kr & 1/90 & $1-\Delta \cdot$ & آمبرزورب بوه (نوعى جاذب سنتتيك) \\
\hline rq & $r / \cdot V$ & $\cdot / \cdot T-T \Delta$ & كربن فعال (مدل فيشر) \\
\hline et & $r / 1$ & $\cdot / \xi-r \ldots$ & فيلترازورب · • (نوعى جاذب سنتتيك) \\
\hline 4) & $r / \Lambda$ & $\cdot 11 \cdot 9-1 \cdot \Delta$ & اويتى يور سو LFq (نوعى جاذب سنتتيك) \\
\hline Fa & $r / \cdot 9$ & $.1 \cdot 4-94$ & اويتى يور سو LF (نوعى جاذب سنتتيك) \\
\hline il & 1/9V & $\cdot / \mid \Delta \Delta-\| T \Delta$ & آمبرليت YAD Y (نوعى جاذب سنتتيك) \\
\hline 4) & $\cdot 111$ & $\cdot / r \cdot 9-14 q$ & آمبرليت XAD V (نوعى جاذب سنتتيك) \\
\hline 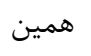 & $1 / 48$ & $1-r \cdot$ & SMZ\#3(زئوليت اصلاح شده با سورفكتانت) \\
\hline مقاله & & & \\
\hline
\end{tabular}

شده با HDTMA داراى راندمان بيشترى نسبت به

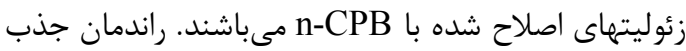

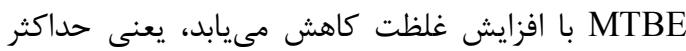
راندمان جذب در غلظتهاى كم صورت مى كيرد و بالعكس،

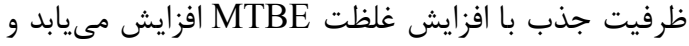
بيشترين ظرفيت جذب در غلظت هاى بالاتر اتفاق

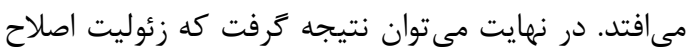
شده با سورفكتانت به علت قيمت بسيار كم و قابليت نقيت كرقيت

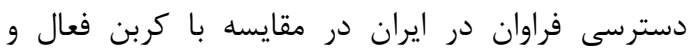

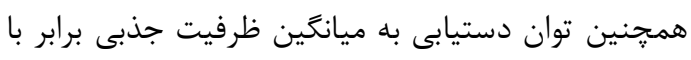

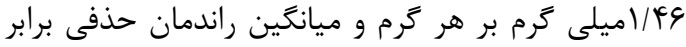

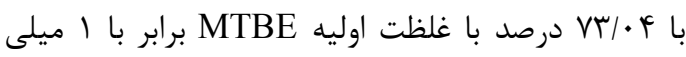

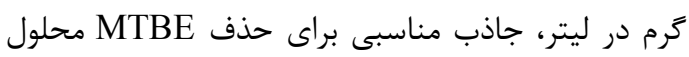

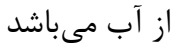$$
\text { تشكر و قدردانى ابل }
$$

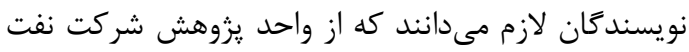

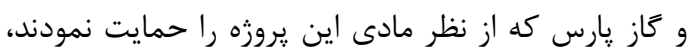

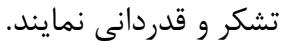

دام مى افتند كه اين مورد با نتايج مطالعات قبلى [FV،FC] نتايج بيان كننده اين مسئله مىباشند كه حذف MTBE

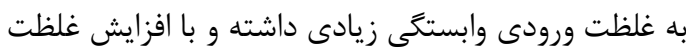
MTBE راندمان حذف در غلظت هاى اوليه پايين تر حاصل مانى

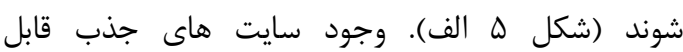
دسترس به اندازه كافى در غلظت هاى كم دليل اصلى بالا

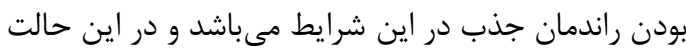

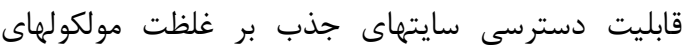

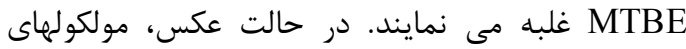

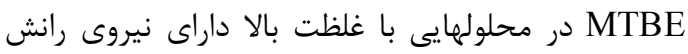
بيشترى بوده و بر نيروى مقاومت انتقال جرم بين فاز داز فيرول جامد و محلول غلبه مينمايد. بنابراين همانطور كه در برد

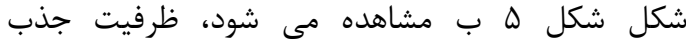
MTBE با افزايش غلظت اوليه افزايش مى يابد. مئ شود،

\section{نتيجه كيرى}

در اين تحقيق مشاهده شد كه زئوليتهاى اصلاح شده به

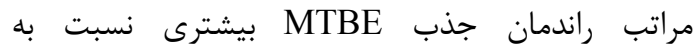

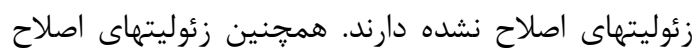




\section{References}

1. Mortazavi S.B, Nikpey A, Rezaee A, Asilian H, Khavanin A, Kazemian H, Methil Tert-Butyl Ether (MTBE) degradiation by a microbial consortium, American journal of environmental sciences 2005; 1 (1):69-73.

2. Mackay D, Shiu W, Ma K, Illustrated handbook of physical-chemical properties and environmental fate for organic chemicals - volatile organic chemicalsChelsea, MI: Lewis, 1993.

3. Klinger J,Stieler C,Sacher F,Branch H.J, MTBE (methyl tertiary-butyl ether) in groundwaters: monitoring results from Germany, J. Environ. Monit 2002 ;4 (2), 276-279.

4. Schmidt T.C, Schirmer M, Weiss H, Haderlein S.B, Microbial degradation of methyl tert-butyl ether and tertbutyl alcohol in the subsurface, J. Contam. Hydrol 2004; 70 (3-4), 173-203.

5. Rubin E, Ramaswami A, The Potential For Phytoremediation Of MTBE,Wat. Res2001; Vol. 35, No. 5, Pp. $1348 \pm 1353$.

6. Deeb R.A, Chu K, Shih T, Linder S, Suffet I, Kavanaugh M.C, MTBE and other oxygenates: environmental sources, analysis, occurrence, and treatment, Environ. Eng. Sci 2003; 20 (5), 433-447. 7. Masoudi-Nejad M, Khatibi M, Analysis of methyl tert-butyl ether in groundwater pollution in Tehran in 2002, Journal of Shahid Beheshti University of Medical Sciences, Tehran University of Medical Sciences 2004;43:11-7[Persian]

8. Kaykhaii M, Mirbaloochzahi M.R, Direct screening of ground water samples for fuel oxygenates by headspace liquid phase microextraction-gas chromatography, Environmental Monitoring and Assessment 2008; 147: 211- 222.

9. Eslami A, Nasseri S, Yadollahi B, Mesdaghinia A, Vaezi F, Nabizade R, Nazmara S, Photocatalytic degradation of methyl tert-butyl ether (MTBE) in contaminated water by $\mathrm{ZnO}$ nanoparticles, Journal of Chemical Technology and Biotechnology 2008; 83: 1447-1453[Persian]

10. Leitner N.K.V, Papailhou A.L, Croue J.P, Peyrot J, Dore M, Oxidation of methyl tert-butyl ether by ozone and combined ozone hydrogenperoxide, Ozone Sci. Eng 1994; 16 (1), 41-54.

11. Mormile M.R, Liu S, Suflita J.M, Anaerobic biodegradation of gasoline oxygenates: extrapolation of information to multiple sites and redox conditions, Environ. Sci. Technol 1994; 28 (9), 1727-1732.

12. Liang S, Palencia L.S, Yates R.S, Davis M.K, Bruno J.M, Wolfe R.L, Oxidation of MTBE by ozone and peroxone processes, J. Am. Water Works Assoc 1999; 91 (6), 104-114.

13. Flores A, Stocking A, Kavanaugh M, Synthetic Resin Sorbents. In: Melin, G. (Ed.), Treatment Technologies for Removal of Methyl
Tertiary Butyl Ether (MTBE) from Drinking Water: Air Stripping, Advanced Oxidation Processes, Granular Activated Carbon, Synthetic Resin Sorbents, National Water Research Institute, California 2000; pp. 261-333.

14. Acero J.L, Haderlein S.B, Schmidt T.C, Suter M.J.F, von Gunten U, MTBE oxidation by conventional ozonation and the combination ozone/hydrogen peroxide: efficiency of the processes and bromate formation, Environ. Sci. Technol 2001; 35 (21), 4252-4259.

15. U.S. Environmental Protection Agency (EPA), Drinking Water Advisory: Consumer Acceptability Advice and Health Effects Analysis on Methyl Tertiary-Butyl Ether (MTBE), Report No.; 822-F97-008. EPA 1997.

16. California Code of Regulations, Title 22, Section 64449, January 07, 1999.

17. Quinlivan P.A, Li L, Knappe D.R.U, Effects of activated carbon characteristics on the simultaneous adsorption of aqueous organic micropollutants and natural organic matter, Water Res 2005; 39 (8), 1663-1673.

18. Faghihian H, Kazemian H, Maragheh M.G, Iranian clinoptilolite-rich tuffs for radionuclide removal from water, J. Radioanal 1999; Nucl. Chem., v. 242(2), pp. 491-495[Persian]

19. Faghihian H, Kazemian H, Zeolite -P Synthesized from Clinoptilolite Rich-tuffs as a Potential Material for Removal of $\mathrm{Cs}+, \mathrm{Sr}+, \mathrm{Ba}++$ and $\mathrm{Ca}++$ from Liquid Radioactive Waste, Nuclear Science Journal 2000; vol. 37, No. 3, pp.180187[Persian]

20. Faghihian $H$, Kazemian $H$, Ion exchange of $\mathrm{Pb}^{2+}, \mathrm{Ag}^{+}, \mathrm{Ni}^{2+}$ and $\mathrm{Zn}^{2+}$ in natural clinoptilolite, study of some parameters, Iranian Journal of Science and Technology 2002; Vol. 26 No. A2, pp. 357-361 [Persian]

21. Menhaje-Bena R, Kazemian H, GhaziKhonsari M, Hosseini M , Shahtaheri S.J, Evaluation of some Iranian natural zeolites and their relevant synthetic zeolites as sorbents for removal of arsenate from drinking water, Iranian Journal of Public Health 2004; Vol .33, No. 1, pp. 36-44[Persian]

22. Malherbe R, Fernandez L s L, Colado L , Phsico - chemical properties of natural zeolites used for adsorption of water. In: Ming DW, Munpton FA, Eds. Int, comm, natural zeolite 1995; pp. 299- 308.

23. Shahtaheri S, Kazemian H, Ghazi-Khonsari M, Menhaje-Bena R, Selective Adsorption and Removal of Arsenic Compounds From Drinking Water Using Natural and Synthetic Zeolites, ICTX2004 conference "Exposure Assessment in a Changing Environment" to Utrecht, The Netherlands, 16-18 June 2004, The Premier 
International Journal in Toxicology 2004; Vol. 197, No. 3, June 15, p. 241[Persian]

24. Shahtaheri S.J, Kazemian H, Menhaje-Bena $\mathrm{R}$, Removal of arsenic species from drinking water by Iranian natural and synthetic zeolites, in Studies in Surface Science and Catalysis 2004; 154 B, Pages 1892-1899[Persian]

25. kazemian H, Darybi K, Mallah M.H, Khani M.R, Vitrification of $\mathrm{Cs}$ and $\mathrm{Sr}$ loaded Iranian Natural and synthetic Zeolites, J. Radioanal. Nucl. Chem 2006; Vol 267, No. 1, 219-223[Persian]

26. Kazemian H, Zakeri H, Rabbani M.S ,Cs and $\mathrm{Sr}$ removal from solution using potassium nickel hexacyanoferrate impregnated zeolites, J. Radioanal. Nucl. Chem 2006; Vol. 268, No. 2 231236[Persian]

27. Kazemian H, Mallah M.H, Elimination of $\mathrm{Cd} 2+$ and $\mathrm{Mn} 2+$ from Wastewaters Using Natural Clinoptilolite and Synthetic Zeolite-P, Iranian Journal of chem. and chem. Eng 2006 Vol. 25, No.4, p. 91-94[Persian]

28. Kazemian H, Mallah M. H, Removal of Chromate ion from Synthetic Water Using MCM41/ZSM-5 Composite, Iran. J. Environ. Health. Sci. Eng 2008; Vol. 5, No. 1, pp. 73-77[Persian]

29. Erdem-Senatalar A, Bergendahl J.A, Giaya A, Thompson R.W, Adsorption of methyl tertiary butyl ether on hydrophobic molecular sieves, Environ. Eng. Sci 2004; 21 (6), 722-729.

30. Bi E, Haderlein S.B, Schmidt T.C, Soprtion of methyl tertbutyl ether (MTBE) and tert-butyl alcohol (TBA) to synthetic resins, Water Res 2005; 39, 4164-4176.

31. Hung H.W, Lin T.F, Adsorption of MTBE from contaminated water by carbonaceous resins and mordenite zeolite, J. Hazard. Mater 2006; 135 (1-3), 210-217.

32. Hejazi M, Anvari M,Bentonite And Zeolite, Iranian Geological Servey Publications, 1992[Persian]

33. Aftabi A, Anvari M,Iranian Mines And Metals, Iranian Geological Servey Publications, 1993[Persian]

34. Karapanagioti H.K, Sabatini D.A, Bowman R.S, Partitioning of hydrophobic organic chemicals (HOC) into anionic and cationic surfactantmodified sorbents, Water Research 2005; 39, p.699-709.

35. Zhaohui L , Bowman R , Jones K, Enhanced Reduction of Chromate and Pelletized SurfactantModified Zeolite/Zerovalent Iron, Environ.Sci.Technol 1999; 33 : 4326-4330.

36. Haggerty G.M, Bowman R.S, Sorption of Chromate and other inorganic anions by organo- zeolite, Environmental Science and Technology 1994; 28, p.452-458.

37. Koh S.M , Dixon J.B, Preparation and Application of Organo-minerals as Sorbents of Phenol, Benzene and Toluene, Applied Clay Science 2001; 18, p.111-122.

38. James D, Prikryl And Roberto T, Pabalan; Sorption Of Uranium (6+) And Neptunium(5+) By Surfactant-Modified Natural Zeolites, Mat. Res. Soc. Symp. Proc 1999; 38(1): 556.

39. Ghiaci M, Abbaspour A, Kia R, SeyedeynAzad F, Equilibrium Isotherm Studies for the Sorption of Benzene, Toluene, and Phenol onto Organo-Zeolites and As-Synthesized MCM-41, Separation and Purification Technology 2004; 40, p. 217-229[Persian]

40. Torabian A, Kazemian H, Seifi L, Bidhendi G.N, Ghadiri S.K, Removal of Petroleum Aromatic Hydrocarbons by Surfactant-Modified Natural Zeolite, CLEAN - Soil, Air, Water 2010;38(1) [Persian]

41. Erping B, Stefan B. H, Torsten C, Sorption of methyl tert-butyl ether (MTBE) and tert-butyl alcohol (TBA) to synthetic resins; Water Research 2005; (39) 4164-4176.

42. Davis S.W, Powers S.E, Alternative sorbents for removing MTBE from gasoline-contaminated ground water, J. Environ. Eng 2000; 126 (4), 354 360 .

43. Lin S.H, Wang C.S, Chang C.H, Removal of methyl tert-butyl ether from contaminated water by macroreticular resin, Ind. Eng. Chem. Res 2002; 41 (16), 4116-4121.

44. Anderson M.A, Removal of MTBE and other organic contaminants from water by sorption to high silica zeolites, Environ. Sci. Technol 2000; 34 (4), 725-727.

45. Flores A, Stocking A, Kavanaugh M, Synthetic Resin Sorbents. In: Melin, G. (Ed.), Treatment Technologies for Removal of Methyl Tertiary Butyl Ether (MTBE) from Drinking Water: Air Stripping, Advanced Oxidation Processes, Granular Activated Carbon, Synthetic Resin Sorbents, National Water Research Institute, California 2000; pp. 261-333.

46. Karapanagioti H.K , Sabatini D.A, Bowman R.S, Partitioning of hydrophobic organic chemicals (HOC) into anionic and cationic surfactantmodified sorbents, Water Research 2005; 39, p.699-709.

47. Hornig G, Northcott K, Snape I, Stevens G, Assessment of sorbent materials for treatment of hydrocarbon contaminated groundwater in cold regions, Cold regions science and technology, 2007. 
Original Article

\title{
MTBE adsorption on Surfactant-Modified Zeolites from aqueous solutions
}

\author{
Nabizadeh $R^{1}$, Mahvi A. $H^{2}$, Ghadiri S. $K^{3}$, Nasseri $S^{4}$, Mesdaghinia $A^{4}$, Abouee $A^{5}$ \\ ${ }^{1}$ Associated professor of Environmental Health engineering, Tehran university of Medical Sciences, Tehran, \\ Iran \\ ${ }^{2}$ Assistant professor of Environmental Health engineering, Center for solid Waste Research, Institute for \\ Environmental Research, Tehran University of Medical Sciences, Tehran, Iran \\ ${ }^{3}$ condidate in Environmental health engineering, Tehran university of Medical Sciences, Tehran, Iran \\ ${ }^{4}$ Professor of Environmental Health engineering, Tehran university of Medical Sciences, Tehran, Iran \\ ${ }^{5}$ M.Sc of Environmental Health, North khorasan university of medical science, Bojnurd, Iran
}

*Corresponding Author: Institute for Environmental Research, Tehran University of Medical Sciences, Tehran, Iran

\section{Abstract}

Background \& Objectives: This study evaluated the ability of natural clinoptilolite zeolite in form of Surfactant Modified Zeolites $\left(S M Z_{S}\right)$ in removal of methyl tert-butyl ether (MTBE) from water using batch tests. Direct comparison between none modified zeolite (NMZ) and Surfactant-Modified Zeolites (SMZ) was made for MTBE removal from water.

Materials \& Methods: The two Clinoptilolite samples used in this study were obtained from Semnan and Meyaneh province. Before the synthesis of the surfactant modified zeolites, the two kinds of zeolites were milled and sieved in to two sizes ranging $0.21-0.25 \mathrm{~mm}$ (60 to 70Mesh) as fine and 0.59-0.84mm (20 to $30 M e s h)$ as coarse. Total cation exchange capacity (CEC) and the external CEC (ECEC) of zeolites were measured and the best zeolite according to $C E C \& E C E$ for rest of the examinations was selected.

Results: Results of this study showed that adsorption capacity of the zeolites will be remarkably improved toward MTBE molecules by modification of its surface by surfactants like Hexa Decyl Trimethyl Ammonium-Chloride (HDTMA-CL) \& n-Cetyl Pyridinium Bromide (CPB). Especially the HDTMA had more effective performance and could receive to average adsorption capacity of $13.02 \mathrm{mg} / \mathrm{g}$ and average removal efficiency of 65.11 percent by the initial MTBE concentration of $10 \mathrm{mg} / \mathrm{L}$.

Conclusion: According to the results of this study SMZ are suitable candidate for removal of MTBE molecules from contaminated solutions.

Keywords: Clinoptilolite; Modified Zeolite; Surfactant; MTBE; Adsorption

Submitted:17 Jun 2012

Revised:14 Oct 2012 
